\title{
A Hierarchical Clustering Algorithm Based Computer Aided Molecular Modeling with Haematoxylin \& Eosin Images of Colon Cancer
}

\author{
K. Satish Reddy ${ }^{1}$, Shaik Basha ${ }^{2}$, B. Prashanth ${ }^{3}$, K. Sridhar Reddy ${ }^{4}$ \\ ${ }_{1,2,3,4}$ Department of ECE, Vardhaman College of Engineering, Hyderabad 501218, India.
}

\begin{abstract}
Color Image segmentation plays a crucial role in many medical imaging applications. In this paper we present a novel algorithm for computer aided molecular modeling in MATLAB R2012, a real time simulation environment. This manuscript presents a novel algorithm for color image segmentation of Haematoxilin and Eosin images of colon cancer. With help of this algorithm we can classify the differences between benign and malignant tumor cells.
\end{abstract}

Keywords: - Color segmentation, Hierarchical Clustering, Real Time Simulation Environment, Molecular Modeling.

\section{INTRODUCTION}

In this manuscript we compare the existing k-means clustering based Computer Aided Molecular Modeling approach of H \& E (Haematoxilin \& Eosin) images of Colon Cancer as illustrated in [1] with the current hierarchical clustering based approach. Ideally image segmentation is defined as the separation of an image into non-overlapping, constituent regions which are homogeneous with respect to some characteristics such as intensity or texture [2]. In present research work, we developed computer image-processing techniques for classifying colon cancer and non-malignant tissue in digital histological images acquired from tissue sections captured using scanning electronic microscope (SEM) acquired with high resolution charged coupled device (CCD) camera from regions of interest (ROI) marked by pathologists on colon tissue sections [5].

\section{DESIGN OF ALgORITHM}

The different steps involved in the design of algorithm are explained as follows:

a. The input color image will be coarsely represented using 25 bins.

b. Coarse representation uses the spatial information from a Histogram based windowing process.

c. Hierarchical clustering is used to cluster the coarse image data.

d. Parameters Used Input Color Image

Number of bins for coarse representation

Window size for histogram processing

Number of classes

Output segmented image

The coding part is illustrated as shown in the below code snippet, this coding part is done in MATLAB R2012 - a real time simulation environment [6].

\%Clear Memory \& Command Window

clc;

clear all;

close all;

\%Parameters for the Segmentation

nBins $=5$;

winSize=7;

nClass $=6$;

$\%$ Read Input Image

$\mathrm{x}=$ imread('DSS.PNG');

figure;imshow(x);title('Input Image');

$\%$ Segmentation

outImg = colImgSeg $(\mathrm{x}, \mathrm{nBins}$, winSize, $\mathrm{nClass})$;

$\%$ Displaying Output 
figure;imshow(outImg);itle('Image labled by cluster index');

\%colormap('default');

\%Image segmentation using Hierarchical clustering algorithm

$\mathrm{xr}=\mathrm{x}(:,:, 1)$;

$\mathrm{xg}=\mathrm{x}(:,,:, 2)$

$\mathrm{xb}=\mathrm{x}(:,,:, 3)$;

figure, imshow(xr, [ ]);itle('objects in cluster 1');

figure, imshow (xg, [ ]); title('objects in cluster2');

figure, imshow (xb, [ ]);itle('objects in cluster3');

se = strel('disk', 3);

$\mathrm{f}=\operatorname{imopen}(\mathrm{xg}, \mathrm{se})$;

\%figure, imshow(f, [ ]);

$\operatorname{xgd}=$ im2double(f);

$\mathrm{w}=$ fspecial('average');

xgd2 = imfilter(xgd, w, 'replicate');

\%figure, imshow (xgd2, [ ]);

wg = fspecial('gaussian',[9 9], 1.8);

xgd3 = imfilter(xgd2, wg,'conv', 'replicate');

\%figure ,imshow(xgd3, [ ]);

$\mathrm{wm}=$ fspecial('average',[69 69]);

$\mathrm{xgb}=$ imfilter( $\mathrm{xgd} 3$,wm, 'replicate');

\% figure, imshow(xgb, [ ]);

$\mathrm{d}=255^{*} \times g \mathrm{~d} 3-255^{*} \mathrm{xgb}$

\%figure ,imshow(d, [ ]);

dmat $=$ mat2gray $(\mathrm{d})$;

isc $=$ im2uint8(dmat);

\% figure ,imshow(isc, [ ]);

ginmax $=\max (\max ($ isc $))$;

$[\mathrm{m}, \mathrm{n}]=\operatorname{size}(\mathrm{isc})$;

giimax $=\max ($ isc $)$;

for $\mathrm{i}=1: \mathrm{m}$

for $\mathrm{j}=1: \mathrm{n}$

$\mathrm{g}=\operatorname{double}(\mathrm{isc}(\mathrm{i}, \mathrm{j}))+128$-ginmax;

if $g<0$

ih $(\mathrm{i}, \mathrm{j})=0$;

elseif $\mathrm{g}>255$

$\mathrm{ih}(\mathrm{i}, \mathrm{j})=255$;

else

$\mathrm{ih}(\mathrm{i}, \mathrm{j})=\mathrm{g}$;

end

end

end

figure ,imshow(ih, [ ]);

title('blue nuclei');

Modeling steps involved are from reading the image to segmentation of the nuclei into a separate image [3].

Further there are also intermediate steps that are involved between reading the image and segmenting the nuclei into a separate image in MATLAB real-time simulation environment [4]. 
Flow chart is designed as follows

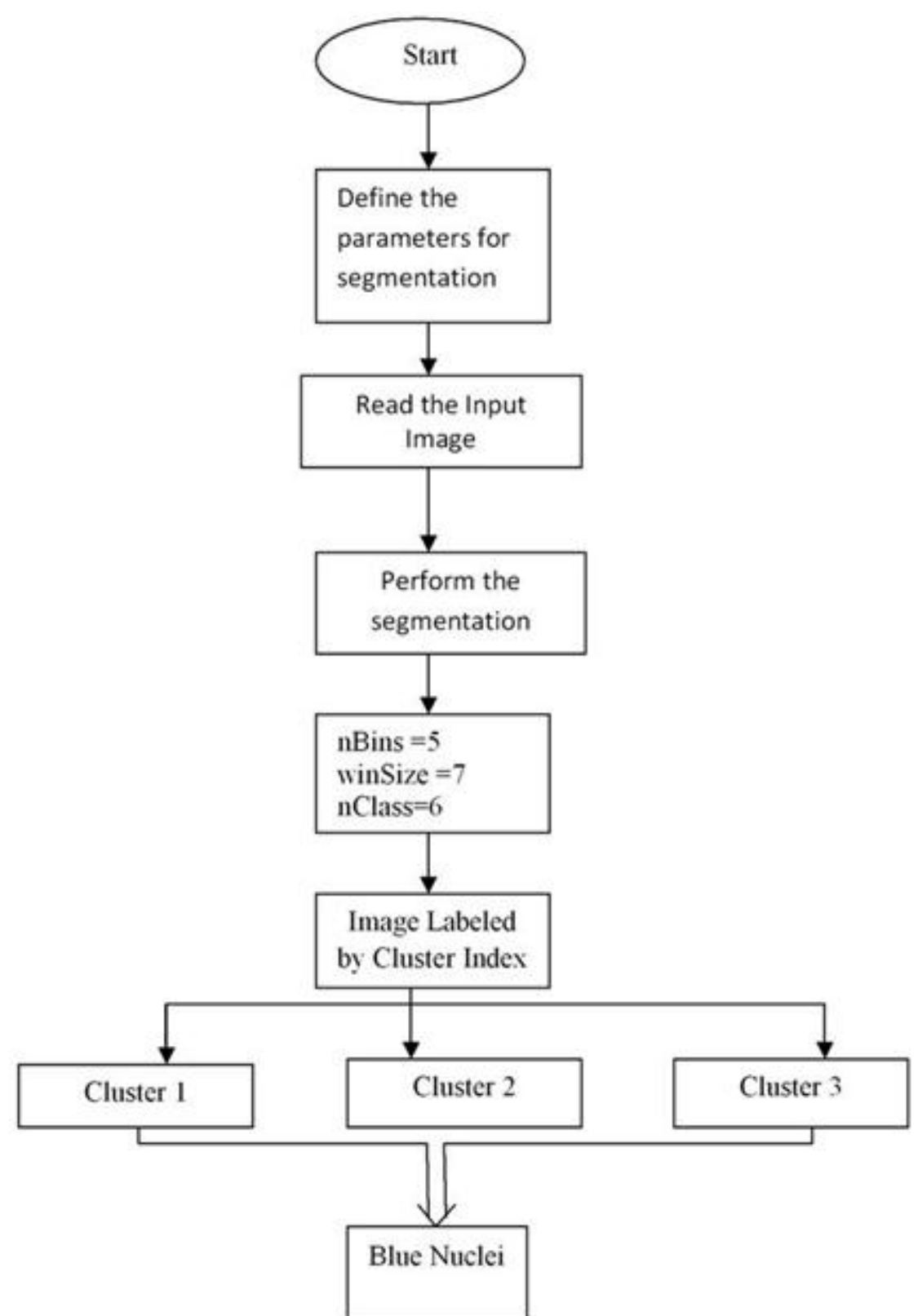

Final DNA Rich Structure

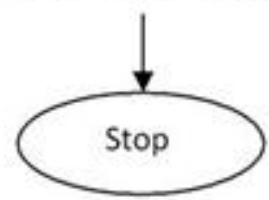




\section{RESULTS AND DISCUSSIONS}

The resulting output flow diagram as shown in Figure 1 is derived from the algorithm based on the above steps.

Inqut lmage

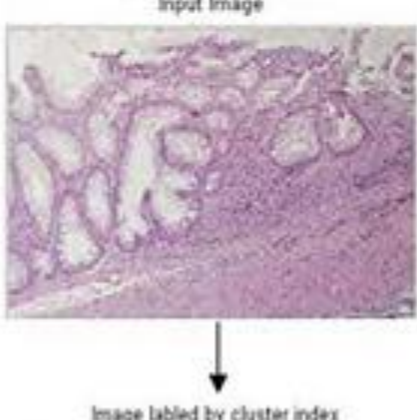

mage buled by chater inder
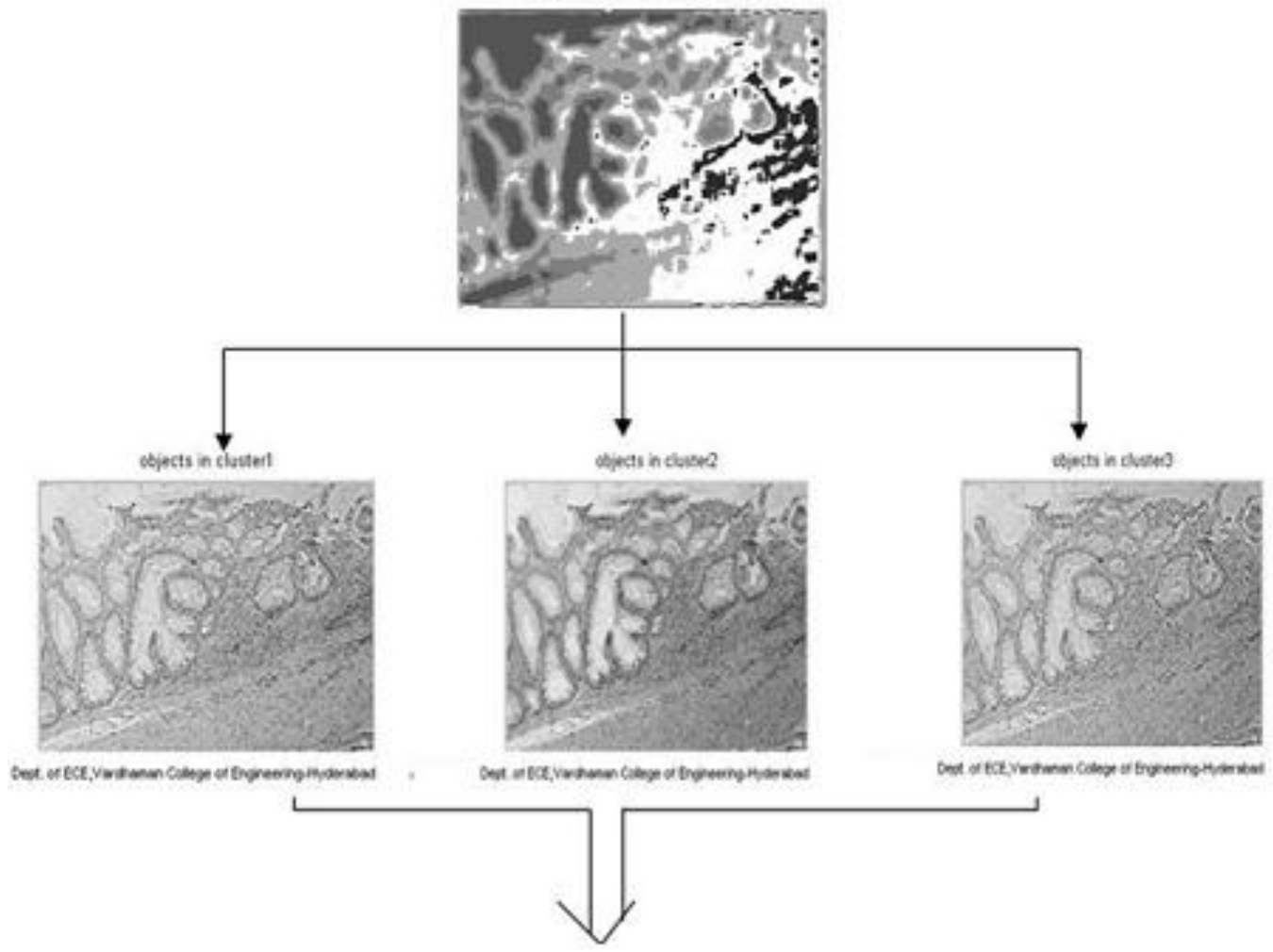

Moe noclei

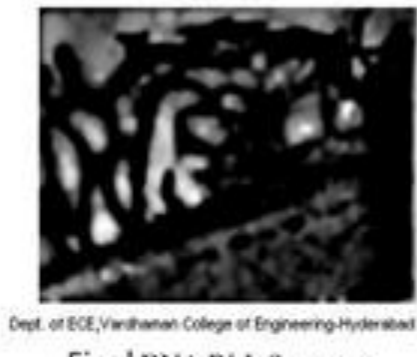

Final DNA Rich Structure

Figure 1: Output Results

\section{CONCLUSIONS}

We have proposed a novel algorithm for color segmentation based on hierarchical clustering. Our algorithm improves the color segmentation accuracy more significantly than previous approaches. One distinctive advantage of our approach is that our algorithm also provides an explicit feature for ROI which can be used for classification and recognition of benign and malignant tissues. 


\section{REFERENCES}

[1] Venkata Subbaiah Kotakadi, Gaddam Susmila Aparna, Prashanth B. U. V. and Sai D. V. R. Gopal. Article: Computer Aided Molecular modeling approach of $\mathrm{H} \& \mathrm{E}$ (Haemotoxylin \& Eosin) images of Colon Cancer. International Journal of Computer Applications 44(9):5-8, April 2012. Published by Foundation of Computer Science, New York, USA.

[2] Otsu Nobuyuki, “A Threshold Selection Method from gray level Histograms”, vol. SMC-9, no.1, January 1979.

[3] G.Deng, L. W. Cahill and G.R. Tobin, "The Study of Logarithmic Image Processing Model and Its Application to Image Enhancement".

[4] Joongho Chang, Gunhee Han, Hose M. Valverde, Norman C. Grisworld, J. Francisco Duque-Carillo, Edgar anchez-Sinencio,( 1997) "Cork Quality Classification System using a Unified Image Processing and Fuzzy-Neural Network Methodology", vol. 8, no. 4, July.

[5] Crabb D.P., Edgar D.F., Fitzke F.W., Mcnaught A.I. \& Wynn H.P. (1995). New Approach to Estimating Variability in Visual-Field Data Using An Image-Processing Technique. British Journal of Ophthalmology 79:213-217.

[6] R.C Gonzalez and R.E Woods , "Digital Image Processing using MATLAB", Addison Wesley/ Pearson education,2nd Education, 2002 . 